\title{
Identification of Domains of Netrin UNC-6 that Mediate Attractive and Repulsive Guidance and Responses from Cells and Growth Cones
}

\author{
Yoo-shick Lim and William G. Wadsworth \\ Department of Pathology, Robert Wood Johnson Medical School, Piscataway, New Jersey 08854-5635
}

Netrin UNC-6 is a protein secreted from ventral cells that guides cell and growth cone migrations in Caenorhabditis elegans. Previously it was shown that UNC-6 domain V-2 regulates dorsal guidance activity and domain $\mathrm{C}$ regulates an activity that prevents the branching of axons when they respond to the $\mathrm{N}$-terminal domains. Because these results indicate that the biological activities of UNC-6 are mediated through specific domains, we systematically examined each UNC-6 domain for guidance activities. Transgenic animals expressing UNC-6 derivatives with domain deletions and mutants with selective unc- 6 loss-of-function mutations were analyzed. The results indicate that the $\mathrm{VI}, \mathrm{V}-2$, and $\mathrm{V}-3$ domains are primarily required for dorsal migrations and the $\mathrm{VI}$ and $\mathrm{V}-3$ domains are required for ventral migrations. These domains are likely important for responses mediated by the UNC-5 and UNC-40 receptors, respectively. Deletion of V-3 and a V-3 point mutation selectively affect either cell or growth cone migrations, indicating that each migration requires unique interactions with UNC-6. Deletion of domain $\mathrm{VI}$ or of a conserved eight amino acid motif within VI causes loss of all UNC-6 guidance activities, and mutations within domain $\mathrm{VI}$ selectively affect different guidance activities, suggesting that domain $\mathrm{VI}$ regulates each response to UNC-6. We propose that individual UNC-6 domains mediate different signals, which act in parallel to regulate the morphological changes necessary for guidance.

Key words: netrin; UNC-6; C. elegans; axon guidance; cell migration; structure-function
UNC-6/netrins are a family of secreted extracellular proteins that can either attract or repel migrating cells and axons. The attractive or repulsive responses of cells and axons to netrin are dependent on the molecular interactions between netrin and cell surface receptors. In Caenorhabditis elegans, migrating cells and axons express $u n c-5$ and $u n c-40$, genes that are required together with unc-6 to direct circumferential migrations (Hedgecock et al., 1990; Leung-Hagesteijn et al., 1992; Chan et al., 1996). unc-5 directs dorsal migrations, whereas $u n c-40$ plays a role in both dorsal and ventral migrations. UNC-40 is a member of the Deleted in Colorectal Cancer family of transmembrane receptor proteins, which are implicated as netrin receptors (Chan et al., 1996; Keino-Masu et al., 1996; Kolodziej et al., 1996; Fazeli et al., 1997). Members of this family have four immunoglobulin and six fibronectin type III extracellular domains and a cytoplasmic domain with several shared domains. Members of the UNC-5 family of transmembrane receptors are also implicated as netrin receptors (Leung-Hagesteijn et al., 1992; Ackerman et al., 1997; Leonardo et al., 1997). They share a common structure of two immunoglobulin and two thrombospondin type 1 extracellular domains and a cytoplasmic domain with several motifs including ZU-5 and Death Domain.

\footnotetext{
Received March 6, 2002; revised May 1, 2002; accepted May 9, 2002.

This work was supported by National Institutes of Health Grant NS33156. We thank Christopher Quinn for comments on this manuscript, members of the Wadsworth laboratory for helpful discussions, Xing-Cong Ren for assistance with microinjection, the Caenorhabditis Genetics Center for strains, and Joseph Culotti, Edward Hedgecock, Harold Hutter, and David Pilgrim for generously providing GFP marker strains.

Correspondence should be addressed to William G. Wadsworth, Department of Pathology, Robert Wood Johnson Medical School, 675 Hoes Lane, Piscataway, NJ 08854-5635. E-mail: william.wadsworth@umdnj.edu.

Copyright (C) 2002 Society for Neuroscience $0270-6474 / 02 / 227080-08 \$ 15.00 / 0$
}

As with most extracellular matrix and cell adhesion molecules, the netrins comprise multiple modules that are found in functionally divergent proteins. UNC-6 was the founding member of the netrin family, and the domains were designated VI, V-1, V-2, V-3, and C (Ishii et al., 1992). The UNC-6 VI and V modules are homologous to the $\mathrm{N}$ termini of laminin subunits, which are among the most conserved extracellular matrix proteins of basement membranes. UNC-6 C is also a phylogenetically conserved protein module; it has been found in the complement C345 protein family, frizzled related proteins, type I C-proteinase enhancer proteins (PCOLCEs), and tissue inhibitors of metalloproteinases (TIMPs) (Ishii et al., 1992; Leyns et al., 1997; Banyai and Patthy, 1999).

Specific UNC-6 domains appear to regulate different UNC-6 guidance activities (Wadsworth et al., 1996). The analyses of several selective loss-of-function alleles that delete the V-2 coding region indicated that the $\mathrm{V}-2$ module is required for dorsal UNC-6 guidance activity (Wadsworth et al., 1996). Moreover, transgenic rescue experiments suggested that the $\mathrm{C}$ module is required to prevent axons from branching when they respond to the N-terminal domains (Lim et al., 1999; Wang and Wadsworth, 2002). There are at least four distinct responses to UNC-6 that can be separated genetically; these are repulsive and attractive responses and the responses by cells and growth cones (Hedgecock et al., 1990). For these responses, only the V-2 domain is known to play a role, although point mutations within domain V I suggest that this domain also mediates specific responses (Wadsworth et al., 1996). Here we report the results of a systematic analysis of UNC-6 domain functions. The results identify domains required for different guidance responses in vivo, and we propose that UNC-6 domains mediate parallel signals that together direct a guidance response. 


\section{MATERIALS AND METHODS}

C. elegans manipulations. C. elegans cultures were maintained by standard methods (Brenner, 1974). Transformation was performed as described (Mello et al., 1991; Mello and Fire, 1995) by injecting the plasmid construct with the dominant transformation marker pRF4, which contains rol-6(su1006) (Kramer et al., 1990). Strains harboring introduced DNA as an integrated array were constructed by irradiation (Mello and Fire, 1995). Assays were conducted on a sample size of 100 animals from each of at least three independent lines. Behavioral and cellular defects of unc-5, unc-6, and unc-40 mutants were assayed according to the method of Hedgecock et al. (1990). Basic molecular techniques for preparation of plasmid DNA, restriction enzyme digestions, agarose gel electrophoresis of DNA, Western blotting, and other molecular biology methods were by standard methods. Monoclonal antibody 12CA5 (Boehringer Mannheim, Indianapolis, IN) was used to detect UNC-6::HA on Western blots. Transgenic animals containing pIM\#97 sequence express a hemagglutinin (HA) epitope-tagged UNC-6 that is capable of rescuing unc-6 null mutants (Wadsworth et al., 1996).

DNA constructions. The plasmid pIM\#180 was constructed by deleting the eight amino acids $\beta$-like region in domain VI. To make the deletion, two fragments that introduce a HinfI site were PCR-amplified by using pIM\#97 as a template. The primer pair for the first fragment was $5^{\prime}$ GTAGCACCTAGGGTG-3' and 5' -CTTGTAAAGTGCCATTGAATCTGGGAGA- $3^{\prime}$. After the PCR, the PCR-amplified products were digested with SalI and HinfI. The primer sets for the second fragment were 5'TCTCCCAGATTCAATGGCACTTTACAAGACCCCGTTTCAATTCTACTCCTCCGAATGTCG-3' and 5'-GTCAGTTGCGGTGACCCAATCC-3'. For this PCR, the same template was used, and the amplified PCR products were digested with HinfI and SgrAI. A 906 bp fragment was excised with SalI and SgrAI from pIM\#97 and replaced by the above SalI-HinfI and HinfI-SgrAI fragments to generate pIM\#180. The plasmid pIM\#190 was constructed by deleting the whole domain VI. To make the deletion, two fragments that introduce an EcoRI site were PCR-amplified with pIM\#97 as a template. The first fragment was produced by using this primer pair: 5'-CTGTGAGAGAT-CTGGGCC-3' and T7 primer. KpnI and EcoRI cut the PCR-amplified products. The second fragment was amplified using this primer set: 5'-TCGCTGCAAATGTAATGGTC-3' and 5'-GGTACTTGTCTTTC-TTTC-3'. EcoRI and NruI cut this fragment. A 4820 bp fragment was excised with KpnI and NruI from pIM\#97 and replaced with above $K p n I-E c o$ RI and EcoRI-NruI fragment to generate pIM\#190. The plasmid pIM\#206 was constructed by deleting domain $\mathrm{V}-1$. To make this deletion construct, the two fragments that introduce a $S p h$ I site were amplified by PCR with pIM\#97 as a template. For the first fragment, 5'-GAGAACAGACCTTCTGC-3' and 5'-GATCGCATGCACCACCAACTGCC-3' were used as a set of primers. This PCR product was double digested with SgrAI and SphI. For the second fragment, 5'GATCGCATGCGCTTGCAACTGCA-3' and 5'-CACTACAAAAAGGCGCCTGG-3' were used as primers for the PCR with the same template. SphI and NarI were used to digest this amplified DNA. An 1884 bp fragment was excised with SgrAI and NarI from pIM\#97 and replaced by the above SgrAI-SphI and SphI-NarI fragments to generate pIM\#206. The plasmid pIM\#181 was constructed by deleting domain V-2. The pIM\#97 was digested with $X h o I$ and subjected to a Klenow reaction without dNTP. The fragments were separated by agarose gel electrophoresis, and the largest fragment was eluted, digested with NruI, and self ligated to generate pIM\#181. The plasmid pIM\#182 was made by deleting domain V-3. PIM\#97 was partially digested with $X b a I$, a 12375 bp fragment was eluted from the digestion mixture, and this fragment was self ligated to generate pIM\#182. The plasmid pIM\#184 was constructed by replacing domain V-2 with domain V-3. An $X b a I$ fragment (336 bp) containing domain V-3 from pIM\#97 was inserted into $X b a \mathrm{I}$ site of plasmid SK $(+)$. The resulting plasmid was double digested with $P v u \mathrm{II}$ and $X h o \mathrm{I}$, and the $P v u \mathrm{II}-X h o \mathrm{I}$ fragment was inserted into the NruI and XhoI sites of pIM\#97 to generated pIM\#184. The plasmid pIM\#191 was constructed to express only UNC-6 domain $\mathrm{C}$ in ventral nerve cord under the glr-1 promoter. For the first fragment the UNC-6 signal peptide and HA-tag sequences were PCRamplified using pIM\#97 as a template and 5'-GATCGGTACCATGATCAATCAGTATTG-3' and 5'-GTCAGATATCTGAGAGATCTGGG$3^{\prime}$ as primers. This PCR product was double digested with $K p n I$ and $E c o \mathrm{RV}$; the digested fragment was inserted into the $K p n \mathrm{I}$ and EcoRV site of pPD49.26. For the second fragment the UNC-6 domain C sequence was PCR-amplified using pIM\#145 as a template and 5'-GATCGATATCCAGAAATTCCGACC-3' and 5'-GTACGATATCATCTGTGTGACG-3' as primers. This PCR product was digested with EcoRV; the digested fragment was inserted into the EcoRV site of the above constructs. The Bam HI and PstI fragment, which encodes glr-1 promoter sequences, from plasmid PV6 was inserted into BamHI and PstI sites of the above constructs to generate pIM\#191.

Transgenic animals. The strains used in this paper are as follows: IM19: urIs13[IM\#175 pRF4], IM39: urIs13; unc-6(ev400), IM113: urIs62[IM\#97 IM\#175 pRF4]; unc-6(ev400), IM65: urIs13; unc-5(e53), IM167: urIs13; unc-5(e53); unc-6(ev400), IM62: urIs13; unc-40(e1430), IM98: urIs13; unc-40(e1430); unc-6(ev400), IM492: urIs247[IM\#206 pRF4]; edIs20; unc-6(ev400), IM493: urIs248[IM\#206 pRF4]; edIs20; unc-6(ev400), IM494: urIs249[IM\#206 pRF4];edIs20; unc-6(ev400), IM95: urIs56[IM\#181 IM\#175 pRF4]; unc-6(ev400), IM96: urIs57[IM\#181 IM\#175 pRF4]; unc-6(ev400), edIs20[F25B3.3::GFP pRF4], IM513: urIs253[IM\#182 pRF4]; edIs20; unc-6(ev400), IM514: urIs254[IM\#182 pRF4]; edIs20; unc-6(ev400), IM515: urIs255[IM\#182 pRF4]; edIs20; unc-6(ev400), IM522: urIs256[IM\#184 pRF4]; edIs20; unc-6(ev400), IM523: urIs257[IM\#184 pRF4]; edIs20; unc-6(ev400), IM524: urIs258[IM\#184 pRF4];edIs20; unc-6(ev400), IM531: urIs259[IM\#180 pRF4]; edIs20; unc-6(ev400), IM532: urIs260[IM\#180 pRF4]; edIs20; unc-6(ev400), IM533: urIs261[IM\#180 pRF4];edIs20; unc-6(ev400), IM540: urIs262[IM\#190 pRF4];edIs20; unc-6(ev400), IM541: urIs26[IM\#190 pRF4]; edIs20; unc-6(ev400), IM542: urIs264[IM\#190 pRF4]; edIs20; unc-6(ev400), IM545: urIs265[IM\#191 pRF4]; edIs20; unc-6(ev400), IM146: urIs13; unc-6(e78), IM117: urIs13; unc-6(rh204), IM548: edIs20; unc-6(ev436), IM551: edIs20; unc-6(rh46) were used for visualization of all neurons. IM175: rhIs4 [glr-1::GFP], IM202: rhIs4; unc-6(ev400), IM205: rhIs4; unc-5(e53), IM485: rhIs4; unc-5(e53); unc-6(ev400), IM206: rhIs4; unc-40(e1430), IM484: rhIs4; unc-40(e1430); unc-6(ev400), IM486: urIs247[IM\#206 pRF4]; rhIs4; unc6(ev400), IM487: urIs248[IM\#206 pRF4]; rhIs4; unc-6(ev400), IM488: urIs249[IM\#206 pRF4]; rhIs4; unc-6(ev400), IM498: urIs250[IM\#181 pRF4];rhIs4; unc-6(ev400), IM499: urIs251[IM\#181 pRF4]; rhIs4; unc6(ev400), IM500: urIs252[IM\#181 pRF4]; rhIs4; unc-6(ev400), IM507: urIs253[IM\#182 pRF4]; rhIs4; unc-6(ev400), IM508: urIs254[IM\#182 pRF4]; rhIs4; unc-6(ev400), IM509: urIs255[IM\#182 pRF4]; rhIs4; unc6(ev400), IM516: urIs256[IM\#184 pRF4]; rhIs4; unc-6(ev400), IM517: urIs257[IM\#184 pRF4]; rhIs4; unc-6(ev400), IM518: urIs258[IM\#184 pRF4]; rhIs4; unc-6(ev400), IM525: urIs259[IM\#180 pRF4]; rhIs4; unc6(ev400), IM526: urIs260[IM\#180 pRF4]; rhIs4; unc-6(ev400), IM527: urIs261[IM\#180 pRF4]; rhIs4; unc-6(ev400), IM534: urIs262[IM\#190 pRF4]; rhIs4; unc-6(ev400), IM535: urIs26[IM\#190 pRF4]; rhIs4; unc6(ev400), IM536: urIs264[IM\#190 pRF4]; rhIs4; unc-6(ev400), IM543. urIs265[IM\#191 pRF4]; rhIs4; unc-6(ev400), IM203: rhIs4; unc-6(e78), IM204: rhIs4; unc-6(rh204), IM546: rhIs4; unc-6(ev436), IM549: rhIs4; unc-6(rh46) were used for visualization of PVC interneurons. NW1099: evIs82a[unc-129::GFP], IM207: evIs82a; unc-6(ev400), IM210: evIs82a; unc-5(e53), IM261: evIs82a; unc-5(e53); unc-6(ev400), IM211: evIs82a; unc-40(e1430), IM260: evIs82a; unc-40(e1430); unc-6(ev400), IM489: urIs247[IM\#206 pRF4]; evIs82a; unc-6(ev400), IM490: urIs248[IM\#206 pRF4]; evIs82a; unc-6(ev400), IM491: urIs249[IM\#206 pRF4]; evIs82a; unc-6(ev400), IM501: urIs250[IM\#181 pRF4]; evIs82a; unc-6(ev400), IM502: urIs251[IM\#181 pRF4]; evIs82a; unc-6(ev400), IM503: urIs252[IM\#181 pRF4]; evIs82a; unc-6(ev400), IM510: urIs253[IM\#182 pRF4]; evIs82a; unc-6(ev400), IM511: urIs254[IM\#182 pRF4]; evIs82a; unc-6(ev400), IM512: urIs255[IM\#182 pRF4]; evIs82a; unc-6(ev400), IM519: urIs256[IM\#184 pRF4]; evIs82a; unc-6(ev400), IM520: urIs257[IM\#184 pRF4]; evIs82a; unc-6(ev400), IM521: urIs258[IM\#184 pRF4]; evIs82a; unc-6(ev400), IM528: urIs259[IM\#180 pRF4]; evIs82a; unc-6(ev400), IM529: urIs260[IM\#180 pRF4]; evIs82a; unc-6(ev400), IM530: urIs261[IM\#180 pRF4]; evIs82a; unc-6(ev400), IM537: urIs262[IM\#190 pRF4]; evIs82a; unc-6(ev400), IM538: urIs26[IM\#190 pRF4]; evIs82a; unc-6(ev400), IM539: urIs264[IM\#190 pRF4]; evIs82a; unc-6(ev400), IM544: urIs265[IM\#191 pRF4]; evIs82a; unc-6(ev400), IM208: evIs82a; unc-6(e78), IM209: evIs82a; unc-6(rh204), IM547: evIs82a; unc-6(ev436), IM550: evIs82a; unc-6(rh46) were used for visualization of DA and DB motor neurons.

\section{RESULTS}

\section{Mapping domains required for UNC-6 function}

Derivatives of $u n c-6$ were constructed to encode UNC-6 with specific deletions or substitutions (Fig. 1). For each construct, the microinjected DNA was integrated into a chromosome, and three independently derived transgenic lines were established. The proteins were designed to include an N-terminal HA epitope tag, 


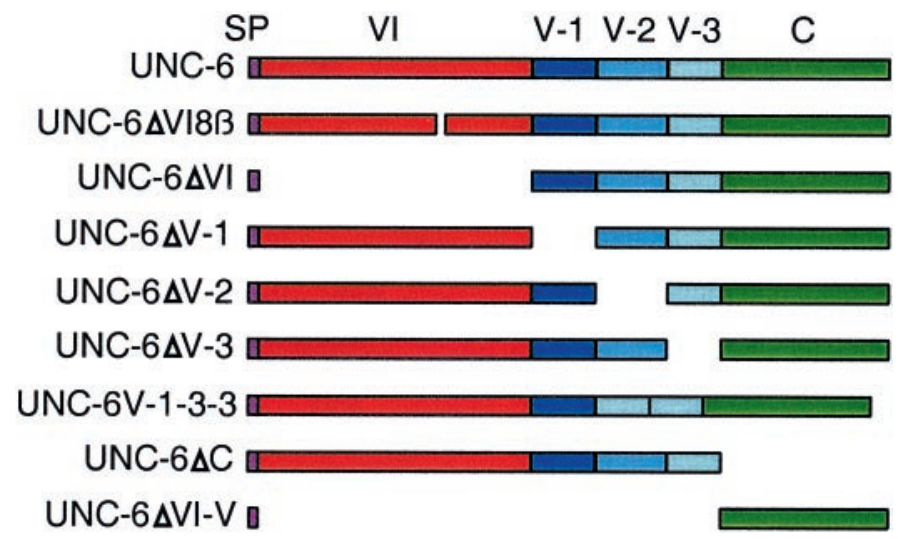

Figure 1. Schematic representation of the netrin UNC-6 protein and UNC-6 derivatives used in this study. UNC-6 comprises an N-terminal domain VI (residues 1-268) similar to the N-terminal domain VI of laminin subunits, three cysteine-rich repeats (residues 269-437) similar to those of domain $\mathrm{V}$ of laminin subunits, and a $\mathrm{C}$-terminal domain named C (residues 438-591) that is not found in laminins but is phylogenetically conserved among other extracellular proteins. An epitope tag comprising three tandem copies of the HA epitope was engineered into a site immediately after the predicted signal peptide $(S P)$.

and the expression of each protein was analyzed by Western blot. The derivatives were tested in the unc- 6 null background for their ability to rescue $u n c-6$ null guidance defects. For each migration assay, 100 animals from each line were scored.

The UNC-6 6 VI construct deletes domain VI. UNC-6/netrin domain VI was named because of its similarity with the N-terminal domain VI of laminin subunits (Ishii et al., 1992). Laminin is a large heterotrimeric extracellular matrix protein formed from three polypeptide chains $(\alpha, \beta$, and $\gamma)$, and in the prototypical laminin each subunit chain contains a domain VI (Timpl and Brown, 1996). Overall, UNC-6 domain VI is most similar to the domain VI of the laminin $\gamma$ subunit; however, a short motif (SADFGKTW) within the UNC-6 domain VI is a hallmark of laminin $\beta$ subunits. Because the phylogenetic conservation of this motif in netrins and laminin $\beta$ subunits suggests a critical function, we specifically deleted these amino acids to create a UNC- $6 \Delta \mathrm{VI} 8 \beta$ derivative.

The UNC-6/netrin V domain was named because of the similarity to the tandem array of cysteine-rich repeats of the $\mathrm{V}-1, \mathrm{~V}-2$, and V-3 motifs of the laminin subunits (Ishii et al., 1992). Overall the UNC- $6 \mathrm{~V}$ domains are most similar to the repeats of the laminin $\gamma$ subunit, with the exception of $\mathrm{V}-2$, which is most similar to the $\mathrm{V}-2$ domain of the laminin $\beta$ subunit. The $\mathrm{V}-2$ repeat is the most highly conserved repeat among the netrins. Within vertebrate netrins, the other laminin-like domains more closely resemble either the laminin $\gamma$ or $\beta$ subunit (Koch et al., 2000). To study the requirement of these domains for UNC-6 guidance, we constructed a series of derivatives that specifically remove each domain, UNC-6 $-6 \mathrm{~V}-1$, UNC-6 $-6 \mathrm{~V}-2$, and UNC-6 $-6 \mathrm{~V}-3$. In addition, we constructed a derivative, UNC-6 V-1-3-3, to test whether the $\mathrm{V}-3$ repeat can functionally substitute for the $\mathrm{V}-2$ repeat.

The third domain of UNC-6 is designated domain C (Ishii et al., 1992). The domain was found at the $\mathrm{C}$ terminus and showed similarity to the $\mathrm{C}$ termini of the complement $\mathrm{C} 345$ protein family. This module was later identified in frizzled related proteins, type I PCOLCEs, and TIMPs (Leyns et al., 1997; Banyai and Patthy, 1999). Interestingly, functionally divergent members of the UNC-6/netrin family have been described that differ

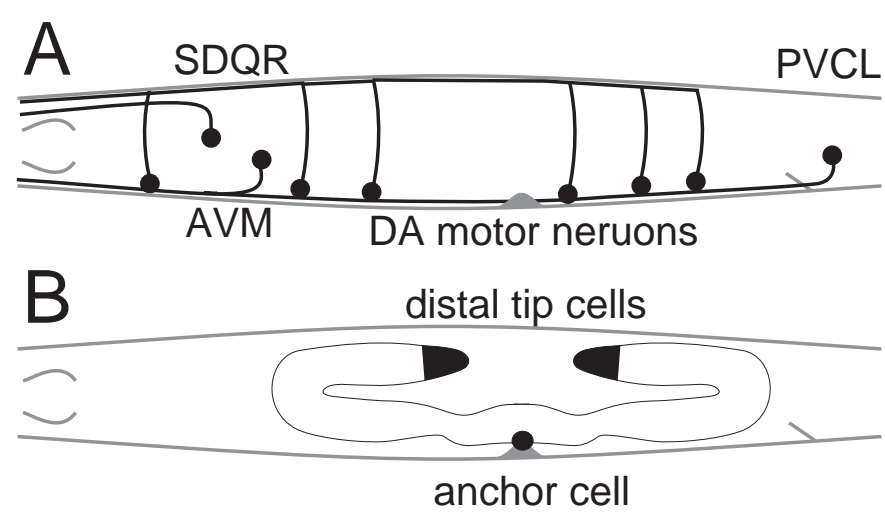

Figure 2. Summary of cell and axon positions. $A$, Cell bodies and axons of representative sensory and motor neurons. The migration of the axons of DA and DB motor neurons and of SDQR were assayed to measure the ability of unc- 6 mutations and transgenes to guide dorsal axon migrations. Only DA motor neurons are represented for simplicity. PVCL and AVM axons were assayed to measure ventral axon migrations. $B$, Migrating mesodermal cells, the distal tip cells, and the anchor cell were assayed to measure dorsal and ventral cell migrations, respectively. Details of the phenotypes of these cells in unc-5, unc-6, and unc-40 mutants were reported by Hedgecock et al. (1990). Anterior is to the left; dorsal is at top.

mainly in the similarity between $\mathrm{C}$ domains (Nakashiba et al., 2000, 2002; Yin et al., 2002). To examine the role of UNC-6 C we constructed the derivative UNC-6 $\Delta$ C. In addition, we investigated the effect that the expression of the $\mathrm{C}$ module alone would have by constructing a UNC-6 $\Delta$ VI-V derivative using a $g l r-1$ promoter to drive expression in ventral nerve cord interneurons.

\section{Domains VI, V-2, and V-3 primarily function in dorsal cell and axon guidance}

To determine whether any of the UNC-6 derivatives are capable of directing dorsal axon migrations, we examined the dorsal migrations of the DA and DB motor neuron axons and of the SDQR axon when each UNC-6 derivative was expressed in the unc-6 null background (Fig. 2). To score the DA and DB migrations, we genetically crossed in an unc-129::gfp transgene that allows these neurons to be visualized by epifluorescence microscopy (Colavita et al., 1998). In the wild-type embryo, axons of the DA and DB neurons migrate circumferentially from the ventral midline cell bodies to form the dorsal nerve cord (Fig. 3). In unc-6 null larvae, the axons wander and longitudinally migrate, resulting in $98 \%$ of the axons failing to reach the dorsal midline (Table 1 ). We find that all the axons reach the dorsal cord in unc- $6 \Delta V-1$ animals, and only $9 \%$ failed to reach the dorsal midline in unc-6 $\Delta C$ animals (Table 1) (Lim et al., 1999). Similar results were obtained when the SDQR axon migrations were scored. The SDQR cell body is located along the lateral body wall, and the axon migrates dorsally to the dorsal sublateral nerve (Fig. 2). The axon was visualized by pan-neural expression of green fluorescent protein (GFP) (Kim et al., 1999). Although 91\% of the axons fail to reach the dorsal sublateral nerve in unc- 6 null animals, $3 \%$ fail in $u n c-6 \Delta V-1$ animals and $56 \%$ fail in $u n c-6 \Delta C$ animals (Table 1). In contrast, expression of UNC-6 $\Delta \mathrm{VI}$, UNC-6 $-6 \mathrm{~V}-2$, UNC-6 $\Delta \mathrm{V}-3$, or UNC-6V-1-3-3 fails to rescue dorsal axon migrations (Tables 1,2$)$. These results indicate that the UNC-6 VI, V-2, and V-3 domains are required primarily for the dorsal axon guidance activities of UNC-6.

To measure the ability of the UNC-6 derivatives to guide dorsal cell migrations, we scored the dorsal migration of the two 

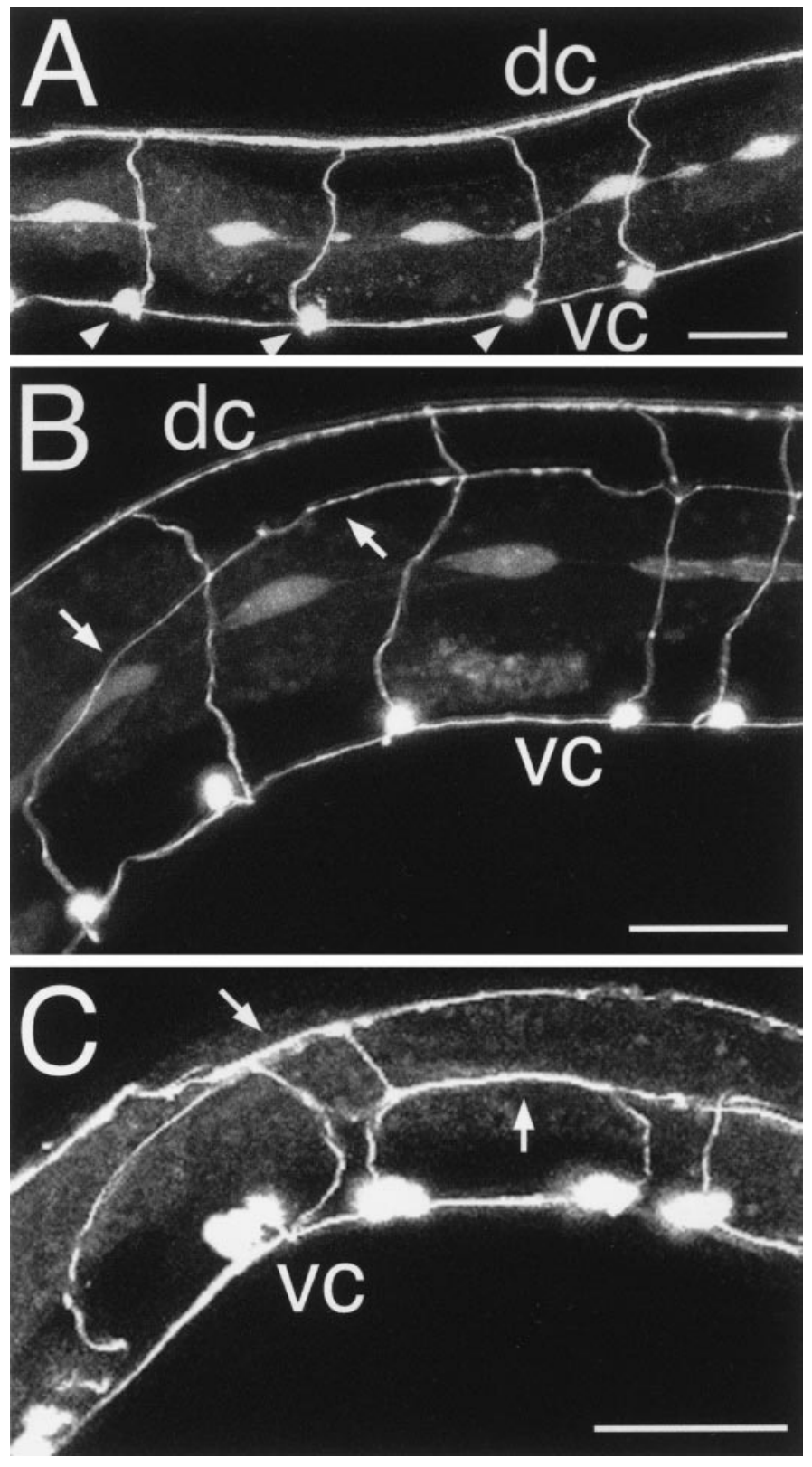

Figure 3. Dorsal axon migrations of DA and DB motor neurons. $A$, In wild-type animals, the DA and DB cell bodies (arrowheads) are positioned along the ventral nerve cord, and each has an axon that migrates longitudinally along the ventral nerve cord $(v c)$ and an axon that migrates circumferentially to the dorsal nerve cord $(d c)$. $B$, In some mutants, the dorsal circumferential migration defects are relatively mild. In this unc-40 mutant, a single axon (arrows) has abnormally migrated at the dorsal sublateral position and fails to reach the dorsal nerve cord. $C$, In unc- 6 mutants, the axons rarely reach the dorsal nerve cord. In this unc- 6 mutant, most axons turn and migrate at the ventral sublateral and lateral positions (arrows). Anterior is to the left; dorsal is at top. Scale bars, $25 \mu \mathrm{m}$.

hermaphrodite distal tip cells (Hedgecock et al., 1990). These cells migrate across muscle and epidermis basement membranes to form the hermaphrodite gonad. At one point, both the anterior and posterior distal tip cells migrate dorsally from their positions along the ventral muscle quadrant (Fig. 2). In unc-6 null animals, $38 \%$ of the anterior and $62 \%$ of the posterior cells fail to migrate dorsally (Table 1). With the exception of UNC-6 $6 \mathrm{~V}-1$ and UNC-
$6 \Delta C$, all of the other UNC-6 derivatives fail to rescue these dorsal cell migrations (Tables 1, 2). All cells migrated normally in unc- $6 \Delta V-1$ animals, and only $3 \%$ of the anterior and $13 \%$ of the posterior cells failed to migrate in $u n c-6 \Delta C$ animals (Table 1) (Lim et al., 1999). These results indicate that the UNC-6 VI, V-2, and V-3 domains are required primarily for the dorsal cell guidance activities of UNC-6.

\section{Domain VI functions primarily in ventral cell and axon guidance, and domain V-3 functions in ventral cell guidance but not ventral axon guidance}

To investigate whether any of the UNC-6 derivatives are capable of directing ventral axon migrations, we examined the ventral axon migrations of the PVCL and AVM axons in the unc-6 null background (Fig. 2). The PVC neurons are in the lateral lumbar ganglia near the tail of the animal; PVCL is in the left lumbar ganglion and PVCR is in the right (Fig. 4). These neurons send axons ventrally along the lumbar commissures in a process that requires the UNC-6-expressing commmissure pioneer PVQ and the UNC-6-expressing midline PVT neuron (Durbin, 1987; Ren et al., 1999). After entering the ventral cord, the PVC axons eventually go the entire length of the cord. To examine the PVC neuron, we used a $g l r-1:: G F P$ transgene that labels 11 interneurons in the ventral nerve cord, the PVC axons being the only ones from the lumbar ganglion (Maricq et al., 1995). We also examined the AVM neuron, which is born in the first larval stage and sends an axon ventrally from the lateral cell body during subsequent developmental stages (Sulston and Horvitz, 1977). The AVM neuron is positioned alone along the right lateral body wall, and its axon migration was scored using pan-neural GFP expression.

In unc- 6 null mutants, $39 \%$ of the PVCL and $27 \%$ of the AVM axons fail to migrate ventrally (Table 1). Expression of UNC$6 \Delta \mathrm{VI}$ or UNC- $6 \Delta \mathrm{VI} 8 \beta$ does not rescue this phenotype, whereas expression of UNC-6 $\Delta \mathrm{V}-1$, UNC- $6 \Delta \mathrm{V}-2$, UNC-6 $6 \mathrm{~V}-3$, UNC$6 \mathrm{~V}-1-3-3$, or UNC-6 $\mathrm{C}$ at least partly rescues these ventral axon migration defects (Tables 1,3 ). These results are in contrast to the dorsal axon migration results, where only UNC-6 $6 \mathrm{~V}-1$ and UNC$6 \Delta \mathrm{C}$ could rescue the unc- 6 null phenotype. Thus, domain VI is required for both dorsal and ventral axon migrations, whereas $\mathrm{V}-2$ and V-3 are required primarily for dorsal axon migrations but not for ventral axon migrations. Domains $\mathrm{V}-1$ and $\mathrm{C}$ are not essential for either dorsal or ventral axon migrations.

To determine whether expression of a UNC-6 derivative affects ventral cell migration, we examined the ability of each transgene to rescue the egg-laying defects of unc- 6 null mutants. In wild-type hermaphrodites, the anchor cell moves to the center of the ventral uterus and induces the ventral epidermal cells to become vulval precursor cells (Fig. 2) (Sternberg and Horvitz, 1986). In the unc-6 null hermaphrodites, the anchor cell is often displaced laterally or dorsally, and the vulva does not develop properly, resulting in animals that are unable to lay eggs (Hedgecock et al., 1990). This occurs in $55 \%$ of the null mutants (Table $1)$. The expression of UNC-6 $6 \mathrm{VI}$, UNC- $6 \Delta \mathrm{VI} 8 \beta$, or UNC$6 \Delta \mathrm{V}-3$ does not rescue the egg-laying defect; however, the expression of UNC- $6 \Delta \mathrm{V}-1$ does rescue and expression of UNC-6 $-6 \mathrm{~V}-2$, UNC-6 V-1-3-3, and UNC-6 $\Delta$ C partly rescues $(34,37$, and $30 \%$ defective, respectively). These results show that the ventral cell migration function requires $\mathrm{VI}$ and $\mathrm{V}-3$ and that $\mathrm{V}-2$ and $\mathrm{C}$ influence the migration but are not essential. Domain V-1 is not required. 
Table 1. Comparison of guidance defects

\begin{tabular}{|c|c|c|c|c|c|c|c|c|}
\hline \multirow[b]{3}{*}{$\begin{array}{l}\text { unc-6 } \\
\text { transgene }\end{array}$} & \multirow[b]{3}{*}{ Genetic background } & \multicolumn{4}{|c|}{ Dorsal guidance ( $\%$ defective) } & \multicolumn{3}{|c|}{ Ventral guidance ( $\%$ defective) } \\
\hline & & \multicolumn{2}{|c|}{ Axon guidance } & \multicolumn{2}{|c|}{ Cell guidance } & \multicolumn{2}{|c|}{ Axon guidance } & \multirow{2}{*}{$\begin{array}{l}\begin{array}{l}\text { Cell } \\
\text { guidance }\end{array} \\
\mathrm{EGL}^{f}\end{array}$} \\
\hline & & $\mathrm{DA}, \mathrm{DB}^{a}$ & $\mathrm{SDQR}^{b}$ & $\begin{array}{l}\mathrm{DTC}^{c} \\
\text { anterior }\end{array}$ & $\begin{array}{l}\text { DTC }^{c} \\
\text { posterior }\end{array}$ & $\mathrm{PVCL}^{d}$ & $\mathrm{AVM}^{e}$ & \\
\hline & Wild type & 0 & $2 \pm 1$ & 0 & 0 & 0 & 0 & 0 \\
\hline \multirow[t]{6}{*}{$u n c-6(+)$} & $u n c-6(e v 400)$ & ND & $3 \pm 1$ & 0 & 0 & ND & 0 & 0 \\
\hline & unc-6(ev400) & $98 \pm 2$ & $91 \pm 3$ & $38 \pm 1$ & $62 \pm 3$ & $39 \pm 2$ & $27 \pm 2$ & $55 \pm 2$ \\
\hline & unc-40(e1430) & $25 \pm 1$ & $57 \pm 4$ & $9 \pm 3$ & $25 \pm 2$ & $26 \pm 1$ & $15 \pm 2$ & $49 \pm 1$ \\
\hline & $u n c-5(e 53)$ & $99 \pm 1$ & $95 \pm 2$ & $40 \pm 3$ & $64 \pm 1$ & 0 & 0 & 0 \\
\hline & unc-40(e1430); unc-6(ev400) & $99 \pm 1$ & $94 \pm 3$ & $43 \pm 3$ & $65 \pm 4$ & $30 \pm 2$ & $25 \pm 3$ & $73 \pm 3$ \\
\hline & unc-5(e53); unc-6(ev400) & $99 \pm 1$ & $93 \pm 4$ & $45 \pm 3$ & $73 \pm 3$ & $35 \pm 2$ & $25 \pm 1$ & $58 \pm 2$ \\
\hline$u n c-6 \Delta V-1$ & unc-6(ev400) & 0 & $3 \pm 1$ & 0 & 0 & 0 & 0 & 0 \\
\hline \multirow[t]{2}{*}{$u n c-6 \Delta V-2$} & $u n c-6(e v 400)$ & $99 \pm 2$ & $85 \pm 3$ & $43 \pm 2$ & $61 \pm 2$ & $5 \pm 2$ & $14 \pm 3$ & $34 \pm 1$ \\
\hline & unc-6(rh204) & $94 \pm 1$ & $88 \pm 4$ & $40 \pm 3$ & $57 \pm 5$ & 0 & $11 \pm 2$ & $16 \pm 2$ \\
\hline unc- $6 V-1-3-3$ & unc-6(ev400) & $98 \pm 3$ & $89 \pm 2$ & $40 \pm 4$ & $64 \pm 4$ & $13 \pm 3$ & $10 \pm 1$ & $37 \pm 3$ \\
\hline \multirow[t]{2}{*}{$u n c-6 \Delta V-3$} & unc-6(ev400) & $95 \pm 2$ & $89 \pm 2$ & $35 \pm 2$ & $65 \pm 3$ & $16 \pm 2$ & $8 \pm 3$ & $50 \pm 3$ \\
\hline & unc-6(e78) & $90 \pm 2$ & $80 \pm 2$ & 0 & $15 \pm 1$ & 0 & 0 & $10 \pm 1$ \\
\hline$u n c-6 \Delta C$ & $u n c-6(e v 400)$ & $9 \pm 2$ & $56 \pm 4$ & $3 \pm 3$ & $13 \pm 2$ & $4 \pm 1$ & $18 \pm 2$ & $30 \pm 2$ \\
\hline
\end{tabular}

${ }^{a}$ The DA, DB motor neuronal cell bodies are located along the ventral nerve cord, and from each an axon migrates dorsally to the dorsal nerve cord.

${ }^{b}$ The SDQR neuron cell body is located laterally, and the axon migrates dorsally to the dorsal sublateral nerve in the larva.

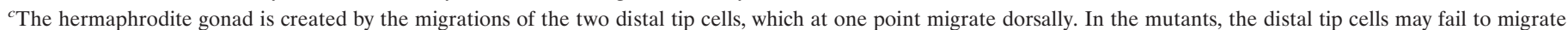
dorsally and instead migrate along the ventral muscle quadrant.

${ }^{d}$ The PVCL neuron cell body is located posteriorly, and the axon migrates ventrally into the ventral nerve cord.

${ }^{e}$ The AVM neuronal cell body is located laterally, and the axon migrates ventrally to the ventral nerve cord.

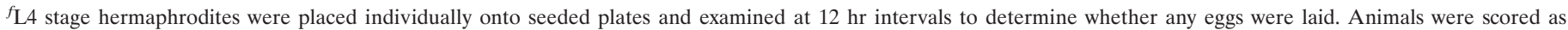
abnormal if they were incapable of egg laying. Three independently derived unc- 6 transgenic strains were counted for each phenotype. $n=100$. Mean \pm SEM.

Table 2. Comparison of dorsal guidance defects

\begin{tabular}{|c|c|c|c|c|c|c|c|c|c|c|c|c|c|}
\hline \multirow{4}{*}{$\begin{array}{l}\text { unc-6 } \\
\text { transgene }\end{array}$} & \multirow[b]{4}{*}{ Genetic background } & \multicolumn{6}{|c|}{ Axon guidance ( $\%$ defective) } & \multicolumn{6}{|c|}{ Cell Guidance (\% defective) } \\
\hline & & \multicolumn{3}{|c|}{ DA, DB } & \multicolumn{3}{|c|}{ SDQR } & \multicolumn{3}{|c|}{ DTC anterior } & \multicolumn{3}{|c|}{ DTC posterior } \\
\hline & & \multicolumn{12}{|c|}{ Temperature $\left({ }^{\circ} \mathrm{C}\right)$} \\
\hline & & 15 & 20 & 25 & 15 & 20 & 25 & 15 & 20 & 25 & 15 & 20 & 25 \\
\hline & unc-6(ev400) & 97 & 97 & 98 & 90 & 91 & 93 & 33 & 38 & 40 & 65 & 62 & 64 \\
\hline & unc-40(e1430) & 23 & 25 & 24 & 54 & 57 & 55 & 8 & 9 & 8 & 20 & 25 & 23 \\
\hline & $u n c-5(e 53)$ & 98 & 99 & 99 & 90 & 95 & 92 & 41 & 40 & 43 & 65 & 64 & 67 \\
\hline & unc-40(e1430); unc-6(ev400) & 99 & 99 & 99 & 92 & 94 & 93 & 42 & 43 & 45 & 66 & 65 & 67 \\
\hline & unc-5(e53); unc-6(ev400) & 98 & 99 & 99 & 95 & 93 & 95 & 46 & 45 & 47 & 74 & 73 & 75 \\
\hline & unc-6(rh46 ev436) & 4 & 10 & 95 & 48 & 63 & 90 & 3 & 8 & 15 & 10 & 36 & 45 \\
\hline & $u n c-6(r h 46)$ & 48 & 94 & 98 & 67 & 93 & 95 & 25 & 44 & 47 & 45 & 75 & 78 \\
\hline$u n c-6 \Delta V I 8 \beta$ & $u n c-6(e v 400)$ & 51 & 98 & 98 & 70 & 90 & 95 & 32 & 41 & 40 & 50 & 73 & 71 \\
\hline$u n c-6 \Delta V I$ & $u n c-6(e v 400)$ & 98 & 98 & 99 & 90 & 93 & 92 & 46 & 45 & 46 & 73 & 72 & 75 \\
\hline
\end{tabular}

\section{Domain $\mathrm{C}$ is not required for guidance activity but inhibits an axon-branching activity}

UNC-6 $\Delta$ C has guidance activity (Lim et al., 1999) (Table 1). In addition, we reported previously that the expression of UNC-6 $\Delta \mathrm{C}$ causes ventral nerve cord motor neurons to extend additional circumferential branches (Lim et al., 1999). Further studies have indicated that the UNC-6 C domain silences ectopic branching, which is dependent on the CaMKII and DAG signaling pathways (Wang and Wadsworth, 2002). We report here that the expression in the ventral nerve cord interneurons of UNC- $6 \Delta \mathrm{VI}-\mathrm{V}$, which comprises only UNC-6 C, does not rescue any of the motor neuron guidance defects in unc-6 null animals, nor are any other abnormalities observed (data not shown). This is consistent with the proposal that the UNC-6 C domain acts in cis to the $\mathrm{N}$-terminal domains to silence the axon (Wang and Wadsworth, 2002).

\section{unc-6 transgenes encoding domain deletions cause phenotypes similar to selective loss-of-function alleles}

A caveat concerning the interpretation of the transgene results stems from the difficulty of proving that the phenotypes are not the result of inappropriate expression. Although Western blot analysis indicates that a protein from each transgene is produced, whether the precise wild-type levels and distribution of each 

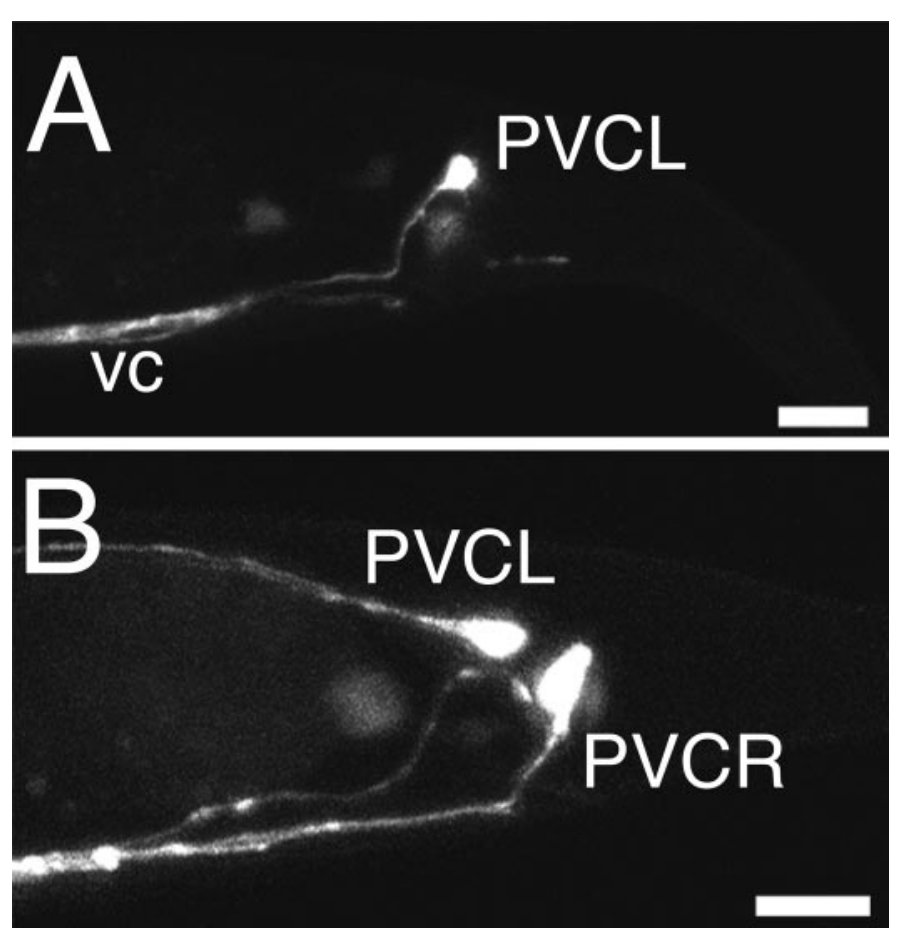

Figure 4. Ventral axon migrations of PVC in the tail region. $A$, In wild-type animals, the PVC neurons extend an axon ventrally along the lumbar commissure to the ventral nerve cord $(v c)$. The left PVC neuron $(P V C L)$ is shown. B, In unc-6 mutants, the PVC axons often migrate at the lateral position instead of directly entering the ventral nerve cord. In this animal, the axon from the left PVC neuron $(P V C L)$ has migrated laterally, whereas the axon from the right PVC neuron (PVCR) has correctly migrated to the ventral nerve cord. Anterior is to the left; dorsal is at top. Scale bars, $10 \mu \mathrm{m}$.

protein are achieved, is difficult to ascertain. Because mutations within the coding sequence of the endogenous gene are more likely to cause phenotypes that result from altered protein structure, we used our assays to examine mutants with selective lossof-function alleles. If individual domains primarily determine a specific guidance activity, then mutations disrupting the coding sequence of that domain might cause some phenotypes that are similar to those caused by the expression of a transgene encoding the protein without that domain. Thus, complementary phenotypes would suggest that a transgene phenotype is the result of altered protein structure rather than inappropriate expression.

We examined four different alleles of unc-6. The first was unc-6(rh204), an allele found previously to contain a deletion that causes the production of a protein lacking the V-2 domain (Wadsworth et al., 1996). Earlier studies indicated that this allele specifically disrupts dorsal cell and axon migrations (Hedgecock et al., 1990). Here we assayed the effects of this mutation by directly scoring axons using neuronal GFP expression. This allows a direct comparison to our transgene expression results. We find that both the unc-6(204) mutation and the expression of the V-2 deletion transgene cause similar phenotypes; that is, dorsal migrations are primarily disrupted (Table 1$)$.

The unc-6(e78) allele was noted previously as having partial gene function (Hedgecock et al., 1990). To quantitate dorsal axon migrations, we scored the DA and DB axons and found that $90 \%$ of the DA and DB circumferential axons failed to migrate dorsally. However, for dorsal cell migrations, $0 \%$ of the anterior and only $15 \%$ of the posterior distal tip cells failed to migrate dorsally
(Table 1). We find that the $e 78$ mutation introduces a tyrosine residue in place of the conserved cysteine at position 410 within domain $\mathrm{V}-3$. These results suggest that a misfolding of the V-3 domain in $u n c-6(e 78)$ mutants does not affect dorsal cell migration functions but does affect dorsal axon migration functions. Both the unc-6(e78) mutation and the expression of the $\mathrm{V}-3$ deletion transgene cause similar phenotypes: disruption of dorsal axon migrations with less affect on ventral axon migrations. Furthermore, both show differences in the ability to guide cell and axon migrations, suggesting that the $\mathrm{V}-3$ domain is responsible for different interactions between UNC-6 and either migrating cells or growth cones.

The unc-6(rh46) and unc-6(rh46 ev436) are alleles that disrupt the coding sequence of the VI domain. The $r h 46$ allele introduces an alanine to proline substitution near the middle of VI, whereas $r h 46 \mathrm{ev} 436$, is a pseudorevertant of $r h 46$ that introduces another substitution, a threonine for an alanine, near the end of VI (Wadsworth et al., 1996). We assayed the effects of these alleles to compare the effects of these mutations with those caused by the expression of VI transgenes. Similar to the phenotypes caused by the expression of the $u n c-6 \Delta V I 8 \beta$ transgene, the $u n c-6(r h 46)$ and unc-6(ev436) mutations also cause a temperature-sensitive loss of guidance activity (Tables 2,3 ). The results from previous assays suggested that the ev436 pseudorevertant restores growth cone but not cell guidance activity (Hedgecock et al., 1990). The results presented here show that the restored activity is heat sensitive. The degree to which different migrations are rescued depends on the temperature at which the assays are performed.

Together these results indicate that point mutations within the coding sequences of the VI, V-2, and V-3 domains each produce unique phenotypes that are similar to those produced by the expression of the transgenes that delete or alter the same domain. This strongly supports the idea that the phenotypes produced by the transgenes are caused by expression of altered protein products.

\section{DISCUSSION}

\section{UNC-6 domains that mediate different guidance functions}

Previous studies indicated that the guidance activities of UNC-6 are mediated through distinct domains (Hedgecock et al., 1990; Wadsworth et al., 1996). Several alleles of unc-6 that cause selective loss of dorsal guidance activity encode for a protein specifically lacking the V-2 domain. Furthermore, two selective loss-of-function alleles that disrupt tissue- and direction-specific guidance were found to be point mutations within domain VI. However, these studies did not show whether the V-2 domain was the only domain required for dorsal guidance, whether other domains are associated with other UNC-6 guidance functions, or how indicative the VI domain point mutations are of domain VI function. The systematic approach taken in this study has addressed these issues and provided evidence that both direction-specific (dorsal versus ventral) and tissue-specific (ectodermal growth cones versus mesodermal cells) guidance requires a combination of different UNC-6 domains.

Dorsal and ventral migrations are mediated by the UNC-6 V-2 and V-3 domains. Deletion of either the V-2 or V-3 domain disrupts dorsal migrations to the same degree as the unc-6(ev400) loss-of-function allele (Table 1). The dorsal guidance activities appear to require the combination of the two domains because substituting a V-3 for the V-2 domain does not restore dorsal 
Table 3. Comparison of ventral guidance defects

\begin{tabular}{|c|c|c|c|c|c|c|c|c|c|c|}
\hline \multirow{4}{*}{$\begin{array}{l}\text { unc- } 6 \\
\text { transgene }\end{array}$} & \multirow[b]{4}{*}{ Genetic background } & \multicolumn{6}{|c|}{ Axon guidance ( $\%$ defective) } & \multirow{2}{*}{\multicolumn{3}{|c|}{$\begin{array}{l}\text { Cell guidance } \\
\text { (\% defective })\end{array}$}} \\
\hline & & \multicolumn{3}{|c|}{ PVCL } & \multicolumn{3}{|c|}{$\mathrm{AVM}$} & & & \\
\hline & & \multicolumn{9}{|c|}{ Temperature $\left({ }^{\circ} \mathrm{C}\right)$} \\
\hline & & 15 & 20 & 25 & 15 & 20 & 25 & 15 & 20 & 25 \\
\hline & unc-6(ev400) & 36 & 39 & 39 & 25 & 27 & 25 & 54 & 55 & 56 \\
\hline & unc-40(e1430) & 23 & 26 & 28 & 16 & 15 & 17 & 48 & 49 & 50 \\
\hline & unc-5(e53) & 0 & 0 & 0 & 0 & 0 & 0 & 0 & 0 & 0 \\
\hline & unc-40(e1430); unc-6(ev400) & 32 & 30 & 35 & 26 & 25 & 27 & 72 & 73 & 75 \\
\hline & unc-5(e53); unc-6(ev400) & 36 & 35 & 39 & 25 & 25 & 23 & 60 & 58 & 61 \\
\hline & unc-6(rh46 ev436) & 0 & 3 & 25 & 0 & 15 & 20 & 15 & 43 & 55 \\
\hline & $u n c-6(r h 46)$ & 20 & 38 & 48 & 13 & 23 & 30 & 32 & 51 & 77 \\
\hline unc- $6 \Delta V 18 \beta$ & $u n c-6(e v 400)$ & 16 & 30 & 59 & 12 & 25 & 32 & 23 & 58 & 80 \\
\hline$u n c-6 \Delta V I$ & unc-6(ev400) & 53 & 55 & 58 & 36 & 35 & 37 & 75 & 78 & 79 \\
\hline
\end{tabular}

guidance functions. The severity of the dorsal guidance defects is similar among unc-5 null mutants and the mutants expressing UNC-6 $-\mathrm{V}-2$, UNC-6 $\mathrm{V}-3$, or UNC-6V1-3-3 (Table 1), suggesting that disrupting the $\mathrm{V}-2$ or $\mathrm{V}-3$ domains interferes with the ability of UNC-6 to interact with the UNC-5 receptor. Among the UNC-6 derivatives, $\Delta \mathrm{V}-3$ most closely resembles the cell and axon ventral defects of the unc-40 mutant (Table 1), suggesting that $\mathrm{V}-3$ is involved in the interaction with UNC-40. Loss of either flanking domain, as in $\Delta \mathrm{V}-2, \Delta \mathrm{C}$, and $\mathrm{V}-1-3-3$ animals, causes a less severe loss of ventral guidance function, suggesting that the flanking domains may influence the interaction between domain V-3 and UNC-40.

The V-3 domain appears to mediate different interactions between UNC- 6 and either migrating cells or growth cones. The unc-6(e78) allele, which disrupts domain V-3, specifically interferes with dorsal axon migrations but not dorsal cell migrations or any ventral migrations (Table 1). Furthermore, deletion of V-3 more strongly disrupts ventral cell migrations than ventral axon migrations. Interestingly, the phenotypes of UNC-5 and UNC-40 mutants do suggest that these receptors mediate responses differently for cells and growth cones. This raises the possibility that unknown cell type-specific UNC-6 receptors associate with the V-3 domain. Alternatively, domain V-3 may influence a difference in the manner in which UNC-6 interacts on cells and growth cones with the UNC-5 and UNC-40 receptors.

Domain VI is essential for all UNC-6 guidance functions. Interestingly, the short but conserved laminin $\beta$ subunit motif within domain VI is critical, suggesting that it may be a site of molecular interaction. Two mutations within domain VI selectively disrupt the guidance activities. The temperature-sensitive unc-6(rh46 ev436) allele selectively restores growth cone migrations, whereas the $u n c-6(e v 400$ ev437) allele, which replaces the domain VI residues glutamine-serine-histidine at positions $78-80$ with two serines, disrupts ventral migrations but not dorsal migrations of both axons and cells (Hedgecock et al., 1990; Wadsworth et al., 1996). The observation that these two alleles selectively disrupt the different guidance activities of UNC-6 provides further evidence that domain VI is required for all guidance activities. It is possible that this domain is involved directly in the association of UNC-6 with UNC-5 and UNC-40. Alternatively, this domain could indirectly affect UNC-5 and UNC-40 signaling through interactions with other receptors and

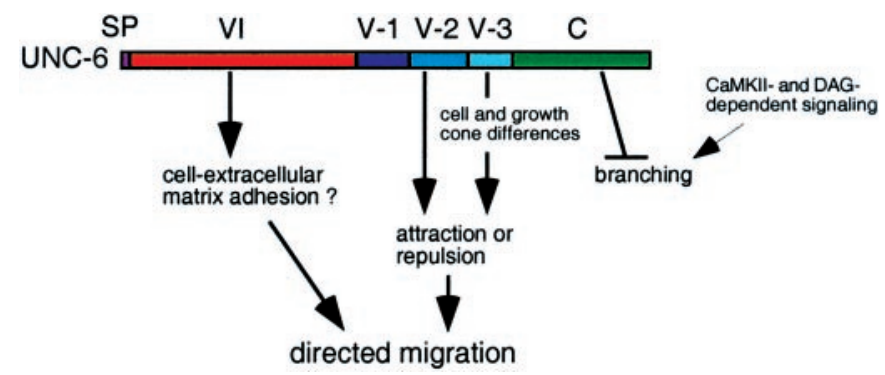

Figure 5. A summary of the proposed function for each of the UNC-6 domains. The combination of domains elicits parallel signals that together mediate the different cytoskeletal changes necessary for guidance.

the extracellular matrix. By influencing properties of cell adhesion, the domain could alter cytoskeletal dynamics that are tied to both UNC-5 and UNC-40 responses.

\section{Model for parallel UNC-6 signaling}

Guidance involves dynamic changes to the cytoskeleton and cell adhesion properties of the migrating cell or growth cone. Our results suggest that the combined activities of different domains mediate UNC-6 guidance (Fig. 5). We propose that domain VI plays a key role in coordinating the cytoskeletal machinery and allowing a response to UNC-6 and that domains V-2 and V-3 elicit signals that specifically direct attraction or repulsion. Finally, the $\mathrm{C}$ domain provides a signal that prevents axon branching in response to the signals elicited by the $\mathrm{N}$-terminal domains (Lim et al., 1999; Wang and Wadsworth, 2002). In this model, the modular nature of the UNC-6 molecule is associated with the parallel signals, and the combination of UNC-6 modules regulates multiple aspects of the guidance machinery.

Our results form the basis for further biochemical studies to investigate molecular interactions between UNC-6/netrin and its receptors. Two UNC-6 receptors are known: UNC-5 mediates repulsion, i.e., migration away from UNC-6 sources, whereas UNC-40 is involved in both attraction and repulsion (Hedgecock et al., 1990). For the repulsive response, it is thought that UNC-5 and UNC-40 can act either independently or together through the formation of UNC-5 or UNC-5 and UNC-40 receptor complexes (Merz et al., 2001). Studies in vitro support such receptor interactions (Hong et al., 1999). We speculate that these receptor 
complexes form primarily through an association with the V-2 and V-3 domains. Although our results also support the possibility that domain VI is involved, we favor the possibility that this domain interacts with other receptors and the extracellular matrix to mediate cytoskeletal and adhesion changes necessary for the UNC-5 and UNC-40 responses. Finally, our results indicate that domain $\mathrm{V}-3$ is responsible for a difference between the ability of cells and growth cones to respond to UNC-6. The molecular explanation for this difference also requires further research.

\section{REFERENCES}

Ackerman SL, Kozak LP, Przyborski SA, Rund LA, Boyer BB, Knowles BB (1997) The mouse rostral cerebellar malformation gene encodes an UNC-5-like protein. Nature 386:838-842.

Banyai L, Patthy L (1999) The NTR module: domains of netrins, secreted frizzled related proteins, and type I procollagen C-proteinase enhancer protein are homologous with tissue inhibitors of metalloproteases. Protein Sci 8:1636-1642.

Brenner S (1974) The genetics of Caenorhabditis elegans. Genetics 77:71-94

Chan SS, Zheng H, Su MW, Wilk R, Killeen MT, Hedgecock EM, Culotti JG (1996) UNC-40, a C. elegans homolog of DCC (Deleted in Colorectal Cancer), is required in motile cells responding to UNC-6 netrin cues. Cell 87:187-195.

Colavita A, Krishna S, Zheng H, Padgett RW, Culotti JG (1998) Pioneer axon guidance by UNC-129, a $C$. elegans TGF-beta. Science 281:706-709.

Durbin R (1987) Studies on the development and organisation of the nervous system of Caenorhabditis elegans. PhD thesis, University of Cambridge.

Fazeli A, Dickinson SL, Hermiston ML, Tighe RV, Steen RG, Small CG, Stoeckli ET, Keino-Masu K, Masu M, Rayburn H, Simons J, Bronson RT, Gordon JI, Tessier-Lavigne M, Weinberg RA (1997) Phenotype of mice lacking functional Deleted in colorectal cancer (Dcc) gene. Nature 386:796-804.

Hedgecock EM, Culotti JG, Hall DH (1990) The unc-5, unc-6, and unc- 40 genes guide circumferential migrations of pioneer axons and mesodermal cells on the epidermis in C. elegans. Neuron 4:61-85.

Hong K, Hinck L, Nishiyama M, Poo MM, Tessier-Lavigne M, Stein E (1999) A ligand-gated association between cytoplasmic domains of UNC5 and DCC family receptors converts netrin-induced growth cone attraction to repulsion. Cell 97:927-941.

Ishii N, Wadsworth WG, Stern BD, Culotti JG, Hedgecock EM (1992) UNC-6, a laminin-related protein, guides cell and pioneer axon migrations in C. elegans. Neuron 9:873-881.

Keino-Masu K, Masu M, Hinck L, Leonardo ED, Chan SS, Culotti JG, Tessier-Lavigne M (1996) Deleted in Colorectal Cancer (DCC) encodes a netrin receptor. Cell 87:175-185.

Kim S, Ren XC, Fox E, Wadsworth WG (1999) SDQR migrations in Caenorhabditis elegans are controlled by multiple guidance cues and changing responses to netrin UNC-6. Development 126:3881-3890.

Koch M, Murrell JR, Hunter DD, Olson PF, Jin W, Keene DR, Brunken WJ, Burgeson RE (2000) A novel member of the netrin family, betanetrin, shares homology with the beta chain of laminin: identification, expression, and functional characterization. J Cell Biol 151:221-234.

Kolodziej PA, Timpe LC, Mitchell KJ, Fried SR, Goodman CS, Jan LY,
Jan YN (1996) frazzled encodes a Drosophila member of the DCC immunoglobulin subfamily and is required for CNS and motor axon guidance. Cell 87:197-204.

Kramer JM, French RP, Park EC, Johnson JJ (1990) The Caenorhabditis elegans rol-6 gene, which interacts with the sqt- 1 collagen gene to determine organismal morphology, encodes a collagen. Mol Cell Biol 10:2081-2089.

Leonardo ED, Hinck L, Masu M, Keino-Masu K, Ackerman SL, TessierLavigne M (1997) Vertebrate homologues of C. elegans UNC-5 are candidate netrin receptors. Nature 386:833-838.

Leung-Hagesteijn C, Spence AM, Stern BD, Zhou Y, Su MW, Hedgecock EM, Culotti JG (1992) UNC-5, a transmembrane protein with immunoglobulin and thrombospondin type 1 domains, guides cell and pioneer axon migrations in C. elegans. Cell 71:289-299.

Leyns L, Bouwmeester T, Kim SH, Piccolo S, De Robertis EM (1997) Frzb-1 is a secreted antagonist of Wnt signaling expressed in the Spemann organizer. Cell 88:747-756.

Lim YS, Mallapur S, Kao G, Ren XC, Wadsworth WG (1999) Netrin UNC-6 and the regulation of branching and extension of motoneuron axons from the ventral nerve cord of Caenorhabditis elegans. J Neurosci 19:7048-7056.

Maricq AV, Peckol E, Driscoll M, Bargmann CI (1995) Mechanosensory signalling in $C$. elegans mediated by the GLR-1 glutamate receptor. Nature 378:78-81.

Mello C, Fire A (1995) DNA transformation. Methods Cell Biol 48:451-482.

Mello CC, Kramer JM, Stinchcomb D, Ambros V (1991) Efficient gene transfer in C. elegans: extrachromosomal maintenance and integration of transforming sequences. EMBO J 10:3959-3970.

Merz DC, Zheng H, Killeen MT, Krizus A, Culotti JG (2001) Multiple signaling mechanisms of the unc-6/netrin receptors unc-5 and unc-40/ dcc in vivo. Genetics 158:1071-1080.

Nakashiba T, Ikeda T, Nishimura S, Tashiro K, Honjo T, Culotti JG, Itohara S (2000) Netrin-G1: a novel glycosyl phosphatidylinositollinked mammalian netrin that is functionally divergent from classical netrins. J Neurosci 20:6540-6550.

Nakashiba T, Nishimura S, Ikeda T, Itohara S (2002) Complementary expression and neurite outgrowth activity of netrin-G subfamily members. Mech Dev 111:47-60.

Ren XC, Kim S, Fox E, Hedgecock EM, Wadsworth WG (1999) Role of netrin UNC-6 in patterning the longitudinal nerves of Caenorhabditis elegans. J Neurobiol 39:107-118.

Sternberg PW, Horvitz HR (1986) Pattern formation during vulval development in C. elegans. Cell 44:761-772.

Sulston JE, Horvitz HR (1977) Post-embryonic cell lineages of the nematode, Caenorhabditis elegans. Dev Biol 56:110-156.

Timpl R, Brown JC (1996) Supramolecular assembly of basement membranes. BioEssays 18:123-132.

Wadsworth WG, Bhatt H, Hedgecock EM (1996) Neuroglia and pioneer neurons express UNC-6 to provide global and local netrin cues for guiding migrations in C. elegans. Neuron 16:35-46.

Wang Q, Wadsworth WG (2002) The C domain of netrin UNC-6 silences calcium/calmodulin-dependent protein kinase- and diacylglycerol-dependent axon branching in Caenorhabditis elegans. J Neurosci 22:2274-2282.

Yin Y, Miner JH, Sanes JR (2002) Laminets: laminin- and netrinrelated genes expressed in distinct neuronal subsets. Mol Cell Neurosci 19:344-358. 